\title{
InGaAs Quantum Dots on Cross-Hatch Patterns as a Host for Diluted Magnetic Semiconductor Medium
}

\author{
Teeravat Limwongse, Supachok Thainoi, \\ Somsak Panyakeow, and Songphol Kanjanachuchai \\ Semiconductor Device Research Laboratory (Nanotec Center of Excellence), Department of Electrical Engineering, \\ Faculty of Engineering, Chulalongkorn University, Bangkok 10330, Thailand
}

Correspondence should be addressed to Songphol Kanjanachuchai; songphol.k@chula.ac.th

Received 19 March 2013; Accepted 13 June 2013

Academic Editor: Sudhakar Nori

Copyright (C) 2013 Teeravat Limwongse et al. This is an open access article distributed under the Creative Commons Attribution License, which permits unrestricted use, distribution, and reproduction in any medium, provided the original work is properly cited.

\begin{abstract}
Storage density on magnetic medium is increasing at an exponential rate. The magnetic region that stores one bit of information is correspondingly decreasing in size and will ultimately reach quantum dimensions. Magnetic quantum dots (QDs) can be grown using semiconductor as a host and magnetic constituents added to give them magnetic properties. Our results show how molecular beam epitaxy and, particularly, lattice-mismatched heteroepitaxy can be used to form laterally aligned, high-density semiconducting host in a single growth run without any use of lithography or etching. Representative results of how semiconductor QD hosts arrange themselves on various stripes and cross-hatch patterns are reported.
\end{abstract}

\section{Introduction}

Quantum dots (QDs) are very small regions of homogeneous material with typical size smaller than $100 \mathrm{~nm}$ for semiconductors [1]. There are two main approaches to synthesize QDs: top down and bottom up [2]. The top-down approach starts with a bulk material and scales it down to small feature using lithography and etching. The bottom-up approach starts with atoms or molecules and scales them up by chemi$\mathrm{cal}$ means to the required size. The quantum dots of interests are diluted magnetic semiconductor (DMS) which, as its name suggests, is electrically semiconducting and magnetically active [3]. While their applications in memory technology have not yet been fully realized, it is expected that various forms of DMS structures can be used as magnetic storage dots or read heads in hard disk drives (HDDs). (For examples, see US Patents no. 7249518 sensors based on giant planar hall effect in dilute magnetic semiconductors, no. 5294287 class of magnetic materials for solid state devices, 6999279 perpendicular patterned magnetic media, no. 6947235 Patterned multilevel perpendicular magnetic recording media, and no. 6906879 magnetic recording system with patterned multilevel perpendicular magnetic recording.) The paper is organized as follows. Section 2 reviews the current magnetic media technologies. Section 3 explains how DMS will benefit hard disk-based storage. Section 4 describes how QD semiconducting hosts for DMS are formed using molecular beam epitaxy (MBE), followed by the summary in Section 5 .

\section{Magnetic Media}

A key characteristic of HDDs is storage capacity in gigabyte which is directly proportional to the areal density of the magnetic medium. The areal density in bits per square inch is a product of bits per inch along a recording track times the number of tracks per inch. Smaller magnetic bits will allow the increases in bits/inch and tracks/inch, resulting in increased areal density and storage capacity. One key goal in HDD research is to increase the areal density which has been increasing approximately at an exponential rate. The rapid increase is enabled by improvements in several technologies, mainly read-write heads and magnetic media.

Magnetic media generally contains Co, due to its high magnetocrystalline anisotropy and coercivity, and is alloyed with $\mathrm{Cr}$ and Pt. The magnetic media are in the form of thin film, typically less than $50 \mathrm{~nm}$ [4], deposited on a glass, 
ceramic, or metal substrate by a vacuum deposition process or by sputtering. Information is stored in small magnetic grains $(\mathrm{CoCrPt})$ segregated in an insulator $\left(\mathrm{SiO}_{2}\right)$. The grain size in current disk generation is kept between 10 and $50 \mathrm{~nm}$ and is a competition between fundamental limits and economic considerations: end users want high-capacity disks which drive down the grain size, but smaller grains are unstable as thermal energy can randomly revert magnetization direction and hence can corrupt the stored information. This superparamagnetic limit thus drives up the grain size and keeps it above $10 \mathrm{~nm}$. Because of the randomness of the grain shapes and sizes, each bit written on the disk must cover about 100 grains to ensure reliable storage [5]. Our proposed approach provides a nonrandom semiconducting host (InAs QDs on cross-hatch pattern) that when diluted with a 3d transition element such as Mn can provide a magnetic medium that theoretically takes a single grain to store one bit. The semiconducting host is a prerequisite for diluted magnetic semiconducting media.

\section{Diluted Magnetic Semiconductors}

Diluted magnetic semiconductors are a class of material that exhibits both semiconducting and ferromagnetic properties and can thus provide a platform for data storage and processing in the same material. A DMS is traditionally defined as a diamagnetic semiconductor doped with a few to several atomic per cent of some transition metal with unpaired $\mathrm{d}$ electrons [6]. DMS materials can be realized by alloying a transition metal with III-V binary (GaMnAs, InMnAs, InMnSb, InFeAs, GaCrSb), ternary (GaInMnAs, GaAlMnSb, InMnAsSb, GaCrAsSb), and quaternary (GaInMnAsP, GaAlInMnSb) compounds [7].

One of the most studied DMS systems is $\mathrm{Ga}_{1-x} \mathrm{Mn}_{x}$ As with $0<x<8 \%$ grown using MBE. Despite significant effort, the maximum Curie temperature of bulk ( $\mathrm{Ga}, \mathrm{Mn})$ As is still well below room temperature, $T_{c}<190 \mathrm{~K}$, yet theories predict that at $x>10 \%(\mathrm{Ga}, \mathrm{Mn})$ As can exhibit room temperature ferromagnetism [8]. However, incorporation of $\mathrm{Mn}$ at such high concentration suffers from solid solubility limits and high vapor growth in MBE: Mn tends to agglomerate on the surface giving rise to elemental Mn clusters, as opposed to the desired random incorporation that results in carrier-mediated spin coupling. Migration-enhanced epitaxy may help but requires precise temperature control [8].

MBE is typically used to grow III-V compounds such as GaAs (the host for GaMnAs), InAs (host for InMnAs), and InGaAs (host for GaInMnAs); all are potential hosts for room-temperature DMS materials. Recently, it has been shown that GaMnAs nanodot arrays are a DMS with $T_{c}=$ $140 \mathrm{~K}$ [9]. The synthesis of such GaMnAs quantum dot arrays, however, requires top-down masking technique which creates structural defects and is thus inferior to self-assembled techniques that can grow defects-free QDs, particularly if the technique can grow self-assembled and self-aligned QDs.

\section{Quantum Dots on Cross-Hatch Patterns}

We demonstrated the use of lattice-mismatched InGaAs films grown on GaAs as templates for the growth of self-assembled and self-aligned QDs. By growing InAs QDs on a nonplanar surface, the nucleation positions of the QDs can be controlled. The use of InGaAs/GaAs stripes and cross-hatch patterns (CHPs) as templates for subsequent nucleation of QDs gives rise to laterally aligned QDs. The areal density of the QDs is dictated by the dislocation line densities. This section describes the results and explains the mechanisms related to the growths of QDs, CHPs, and QDs on CHPs, respectively.

4.1. Quantum Dots. When a material with atomic spacing $a_{1}$ is grown on a substrate with a different atomic spacing $a_{0}$, the growth mode will be principally determined by the degree of misfit $\varepsilon \equiv\left(a_{1}-a_{0}\right) / a_{0}$. For thin films with low misfits, the epilayer will grow layer by layer in the Frank-van der Merwe (FM) mode, resulting in smooth films. The growing layer, however, has a different atomic spacing $\left(a_{0}\right)$ from thermal equilibrium $\left(a_{1}\right)$ and is thus under strain. The associated strain energy is contained in this growing layer. As growth continues and the thickness increases, the strain energy will also increase. When the strain energy is too high, the twodimensional (2D) film is unstable and spontaneous formation of three-dimensional (3D) dots will occur, resulting in the reduction of the total energy of the system. The resulting dots are usually small and exhibit quantum behaviors, hence quantum dots. The growth of semiconductor QDs by this mechanism is a well-known phenomenon called StranskiKrastanow (SK) growth mode [10] and has been observed in the growth of several semiconductor QD systems such as InAs on GaAs [11] or Ge on Si [12]. These QDs are defects-free and are electronically and optically active. Quantum effects which include delta function-like density of states and sizedependent electronic levels allow several physical properties to be tuned. Electronic devices that benefit from QDs as the active medium, or as part of the active layer, include singleelectron transistors [13], flash memory [14], and lasers [15]. With transition element incorporation, the magnetic properties of these QDs can also be tuned.

The spontaneous formation of QDs via the SK growth mode is a statistical process; the positions of QDs are random, and an example is shown in the atomic force microscopic (AFM) image of InAs QDs on low-mismatched InGaAs in Figure 1. Though the size of the QDs is quite uniform, the positions are completely random. For QD hosts to be appropriate as a HDD medium, the QDs must not only have high density but they must also line up in accordance with data read-write scheme. For rotating media with information access by head gimbal assemblies (HGA), the QDs should line up radially. For stationary media with information access by multichannel read-write probe matrix, the QDs should line up orthogonally.

4.2. Cross-Hatch Patterns. Cross-hatch patterns are used as templates to influence the QDs to nucleate orthogonally. 


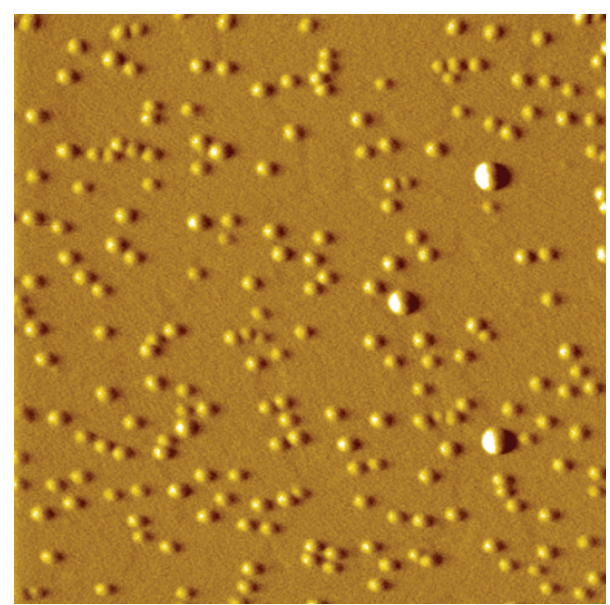

Figure 1: AFM image $\left(2 \times 2 \mu \mathrm{m}^{2}\right)$ of InAs QDs grown on planar InGaAs surface.

CHPs are formed by lattice-mismatched heteroepitaxy similar to the QDs above; the only difference is the degree of mismatch $\varepsilon$. To form a CHP instead of a QD, the misfit needs to be low. For a certain epilayer thickness there is a range of $\varepsilon$ that will result in a cross-hatch pattern.

Figure 2 illustrates the case of lattice-mismatched epitaxy of $50 \mathrm{~nm} \mathrm{In}_{x} \mathrm{Ga}_{1-x}$ As on flat (001)-GaAs substrates. Figure 2(a) shows the primordial stage or the onset of CHP formation at low In molar fraction of $x \approx 8 \%$ or misfit of $\varepsilon \approx 0.573 \%$. The surface is atomically flat: the height contrast in the figure is only $0.79 \mathrm{~nm}$, less than twice the lattice parameters. The AFM image clearly shows atomic steps with dominant surface ripples running along the [110] crystallographic direction. Some ripples running orthogonally, along the [1-10] direction, can be seen to be merging. The ripples along the [1-10] direction merge and form long lines before those along the [110] direction do due to asymmetric adatoms mobilities in the two orthogonal directions which explains the generally observed asymmetric misfit dislocation (MD) line densities.

Figure 2(b) shows the complete CHP formation at In molar fraction of $x=15 \%$ or misfit of $\varepsilon=1.075 \%$. The surface morphology exhibits asymmetrical cross-hatch pattern with MD line density along [1-10] direction greater than [110] direction due to the different formation times as explained above. The heights of most hatches range between 0.5 and $2 \mathrm{~nm}$ with those along the [1-10] direction slightly taller.

There are two challenges in employing CHPs as templates for the growth of regularly arranged, high-density QDs. First, the [110] and [1-10] line densities need be symmetrical. In view of different adatom mobilities as a result of solid sourcederived $\mathrm{As}_{4}$, this asymmetry is fundamental and cannot be avoided. With the availability of a cracker valve or a higher cracking temperature source that yields the more reactive $\mathrm{As}_{2}$ species, the degree of asymmetry is expected to decrease. Second, there is an upper limit to which $\varepsilon$ (or equivalently $x$ ) can be increased before the growth mode changes from $2 \mathrm{D}$ (FM) to 3D (SK). This also sets the upper limit to subsequent QD areal density.
4.3. Quantum Dots on Cross-Hatch Patterns. Growth of InAs QDs on InGaAs CHPs leads to spontaneous alignment of QDs along the cross-hatches. Main parameters used to control/optimize QDs alignment are growth interruption which affects the orderliness of QDs on the hatches [16], crosshatch layer thickness, and composition which affect MD line density [17], growth rates, excess growth, and the use of spacer layer prior to QD growth [18]. The origin and evolution of InAs QD alignment on InGaAs CHPs, which apply to other material systems, are now well understood $[19,20]$. This section captures the main results of our continued efforts towards aligning InAs QDs on InGaAs CHPs.

Figure 3 shows the AFM images of InAs QDs on $50 \mathrm{~nm}$ $\mathrm{In}_{x} \mathrm{Ga}_{1-x}$ As layer grown on (001)-GaAs when the In molar fraction $(x)$ increases from $8 \%$ in Figure $3(a)$ to $10 \%$ in Figure 3(b) and to 15\% in Figures 3(c) and 3(d). The QDs on the surfaces of these samples can be grouped into two broad categories: those nucleated along the buried dislocation lines and those nucleated on the flat regions between the dislocations. For $x=8 \%$, Figure 3(a) shows that the majority QDs are dispersed randomly on the flat regions while the minority formed along the MD lines. The QDs along the MD lines are taller and more closely spaced. For $x=10 \%$, Figure 3(b) shows that the MD line density is greater than that observed in Figure 3(a) and that the proportion of QDs on the MD lines increases as a result. The increased MD line density results from the increased misfit. The greater the misfit the shorter the average dislocation spacing $l$. In the case of $x=8 \%$ in Figure 3(a), only [1-10] MD lines are observed and the average dislocation spacing $l_{[1-10]} \approx 5 \mu \mathrm{m}$. In the case of $x=$ $10 \%$ in Figure $3(\mathrm{~b}), l_{[1-10]} \approx 1.5 \mu \mathrm{m}$ while $l_{[110]}>10 \mu \mathrm{m}$. In the high-misfit case of $x=15 \%$, the dislocation distribution is highly irregular. Some parts of the wafer show moderate MD density as seen in Figure 3(c) while other parts show high or very high density as seen in Figure 3(d). The stochastic nature of dislocation formation gives rise to this asymmetrical behavior, and we believe this particular characteristic is also present in Figures 3(a) and 3(b), although it is possible to tune the growth parameters to obtain dislocation spacing in one direction to be greater than the sample size in which case the surface pattern would not be a cross-hatch one but rather stripes. When the cross-hatch spacing is smaller than adatoms diffusion lengths on the surface, all adatoms will be driven towards the dislocation lines leaving the regions in between completely free of QDs. The denuded zones can be observed in Figures 3(c) and 3(d).

\section{Conclusion}

InAs QDs on InGaAs/GaAs are grown by MBE. Latticemismatched epitaxy results in the formation of QDs, CHPs, stripes, and smooth films in decreasing order of misfit. For low-misfit InGaAs/GaAs, the dislocation lines making up the CHPs are widely spaced, resulting in stripes or crosshatch patterns. For intermediate-misfit InGaAs/GaAs, surface CHPs are characterized by narrowly spaced dislocation lines along the two orthogonal directions with a different line density. Subsequent growth of high-misfit InAs or InGaAs 


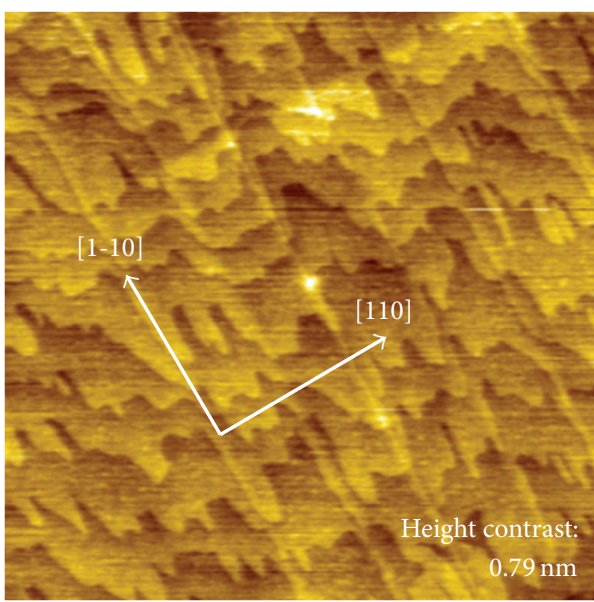

(a)

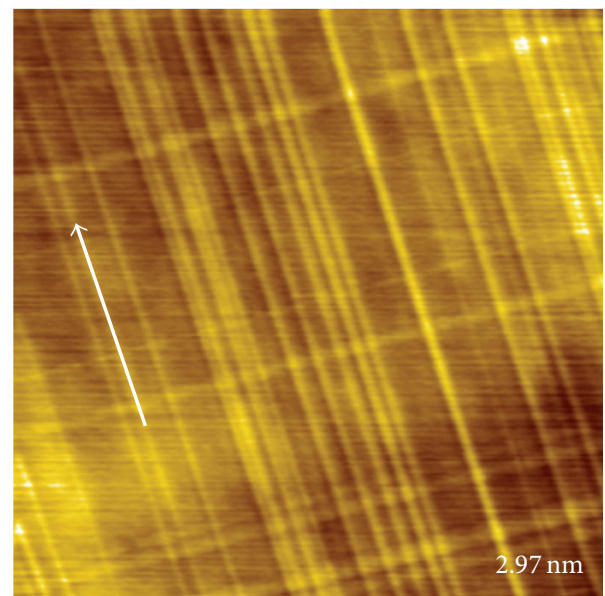

(b)

Figure 2: AFM images of $50 \mathrm{~nm} \operatorname{In}_{x} \mathrm{Ga}_{1-x}$ As films on (001)-GaAs during (a) early or (b) late stages of CHP formation. (a) Image size: $5 \times$ $5 \mu \mathrm{m}^{2}$, height contrast: $0.79 \mathrm{~nm}$, effective $x<8 \%$. (b) Image size: $10 \times 10 \mu \mathrm{m}^{2}$, height contrast: $2.97 \mathrm{~nm}, x=15 \%$.

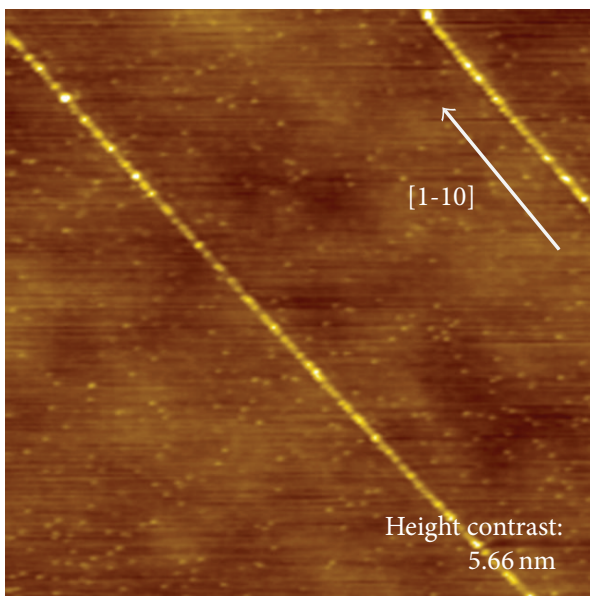

(a)

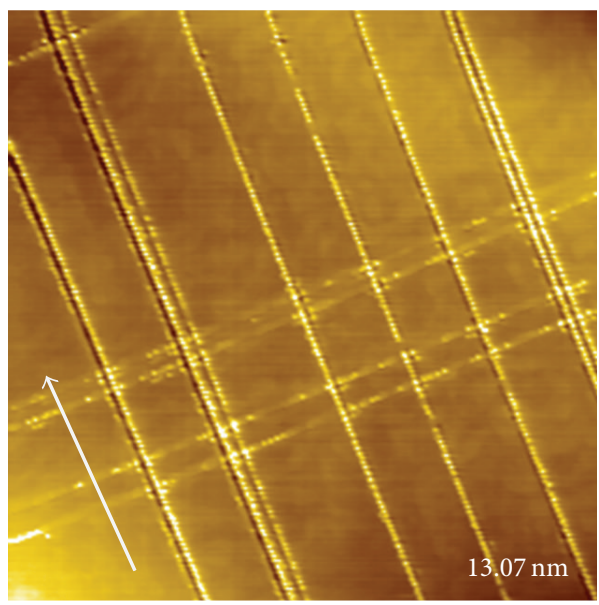

(c)

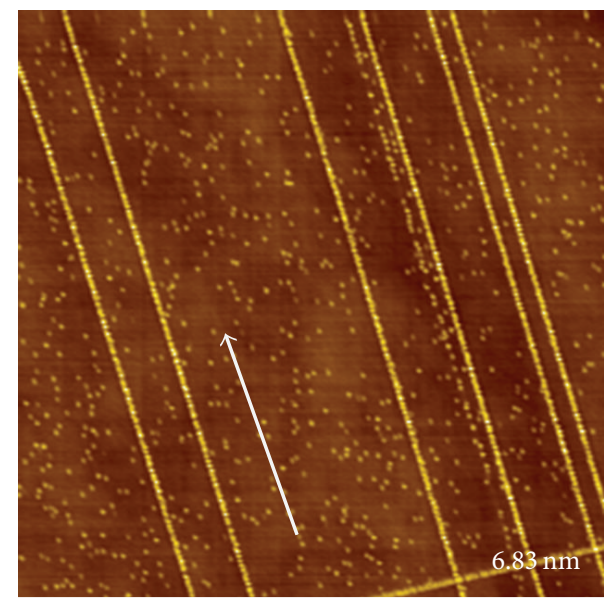

(b)

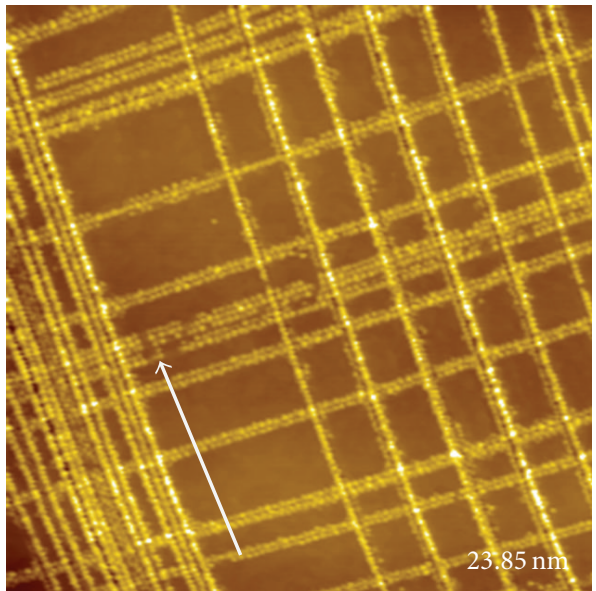

(d)

Figure 3: AFM images $\left(10 \times 10 \mu \mathrm{m}^{2}\right)$ of InAs QDs on $\mathrm{In}_{x} \mathrm{Ga}_{1-x} \mathrm{As} / \mathrm{GaAs}$ surfaces with increasing lattice mismatch, or In molar fraction from (a) $x=0.08$ to (b) 0.10 and (c) and (d) 0.15 . 
on stripes or CHP surfaces results in uni- or bidirectionally aligned QDs, respectively. The unidirectionally aligned QDs result in QD chains with electronic and optical coupling between dots. The bidirectionally aligned QDs result in QD matrices. With appropriate alloying of a transition element and careful control of average dislocation spacings, these QD matrices can exhibit spin coupling and may serve as a highdensity DMS medium/read head.

\section{Acknowledgments}

Pornchai Changmoang is acknowledged for maintaining the MBE system. This work is partially funded by I/UCRC in HDD Component, the Faculty of Engineering, Khon Kaen University (CPN R\&D 01-18-53); NSTDA via Nectec and Nanotec; Thailand Research Fund (DPG5380002, RSA5580015); and the Higher Education Research Promotion and National Research University Project of Thailand, Office of the Higher Education Commission (EN1180A-56).

\section{References}

[1] P. Harrison, Quantum Wells, Wires and Dots: Theoretical and Computational Physics of Semiconductor Nanostructures, John Wiley \& Sons, 2nd edition, 2005.

[2] G. Cao, Nanostructures and Nanomaterials: Synthesis, Properties \& Applications, Imperial College Press, 2004.

[3] M. Jain, Ed., Diluted Magnetic Semiconductors, World Scientific, 1992.

[4] S. O. Kasap, Principle of Electronic Materials and Devices, McGraw-Hill, 3rd edition, 2002.

[5] Perpendicular Magnetic Recording Technologywhite Paper, Hitachi Global Storage Technology, 2011.

[6] S. A. Chambers and B. Gallagher, "Focus on dilute magnetic semiconductors," New Journal of Physics, vol. 10, Article ID 055004, 2008.

[7] L. L. Chang, L. Esaki, H. Munekata, H. Ohno, and S. von Molnar, "Class of magnetic materials for solid state devices," US patent \#5296048.

[8] T. Jungwirth, K. Y. Wang, J. Mašek et al., "Prospects for high temperature ferromagnetism in (Ga,Mn)As semiconductors," Physical Review B, vol. 72, no. 16, Article ID 165204, 2005.

[9] S. P. Bennett, L. Menon, and D. Heiman, "Magnetic properties of GaMnAs nanodot arrays fabricated using porous alumina templates," Journal of Applied Physics, vol. 104, no. 2, Article ID 024309, 4 pages, 2008.

[10] I. N. Stranski and L. Krastanow, "Zur Theorie der orientierten Ausscheidung von Ionenkristallen aufeinander," Sitzungsberichte-Akademie Der Wissenschaften in Wien, MathematischNaturwissenschaftliche Klasse Abteilung IIb, vol. 146, pp. 797810, 1938.

[11] M. Grundmann, O. Stier, and D. Bimberg, "InAs/GaAs pyramidal quantum dots: strain distribution, optical phonons, and electronic structure," Physical Review B, vol. 52, no. 16, pp. 11969-11981, 1995.

[12] F. K. Legoues, M. Copel, and R. M. Tromp, "Microstructure and strain relief of Ge films grown layer by layer on $\mathrm{Si}(001)$," Physical Review B, vol. 42, no. 18, pp. 11690-11700, 1990.
[13] L. Zhuang, L. Guo, and S. Y. Chou, "Silicon single-electron quantum-dot transistor switch operating at room temperature," Applied Physics Letters, vol. 72, no. 10, pp. 1205-1207, 1998.

[14] J. J. Welser, S. Tiwari, S. Rishton, K. Y. Lee, and Y. Lee, "Room temperature operation of a quantum-dot flash memory," IEEE Electron Device Letters, vol. 18, no. 6, pp. 278-280, 1997.

[15] R. Mirin, A. Gossard, and J. Bowers, "Room temperature lasing from InGaAs quantum dots," Electronics Letters, vol. 32, no. 18, pp. 1732-1734, 1996.

[16] C. C. Thet, S. Panyakeow, and S. Kanjanachuchai, "Growth of InAs quantum-dot hatches on InGaAs/GaAs cross-hatch virtual substrates," Microelectronic Engineering, vol. 84, no. 5-8, pp. 1562-1565, 2007.

[17] C. C. Thet, S. Sanorpim, S. Panyakeow, and S. Kanjanachuchai, "The effects of relaxed InGaAs virtual substrates on the formation of self-assembled InAs quantum dots," Semiconductor Science and Technology, vol. 23, no. 5, Article ID 055007, 5 pages, 2008.

[18] S. Kanjanachuchai, M. Maitreeboriraks, C. C. Thet, T. Limwongse, and S. Panyakeow, "Self-assembled InAs quantum dots on cross-hatch InGaAs templates: excess growth, growth rate, capping and preferential alignment," Microelectronic Engineering, vol. 86, no. 4-6, pp. 844-849, 2009.

[19] T. Limwongse, S. Panyakeow, and S. Kanjanachuchai, "Evolution of InAs quantum dots grown on cross-hatch substrates," Physica Status Solidi (C) Current Topics in Solid State Physics, vol. 6, no. 4, pp. 806-809, 2009.

[20] S. Kanjanachuchai and T. Limwongse, "Nucleation sequence of InAs quantum dots on cross-hatch patterns," Journal of Nanoscience and Nanotechnology, vol. 11, no. 12, pp. 10787-10791, 2011. 

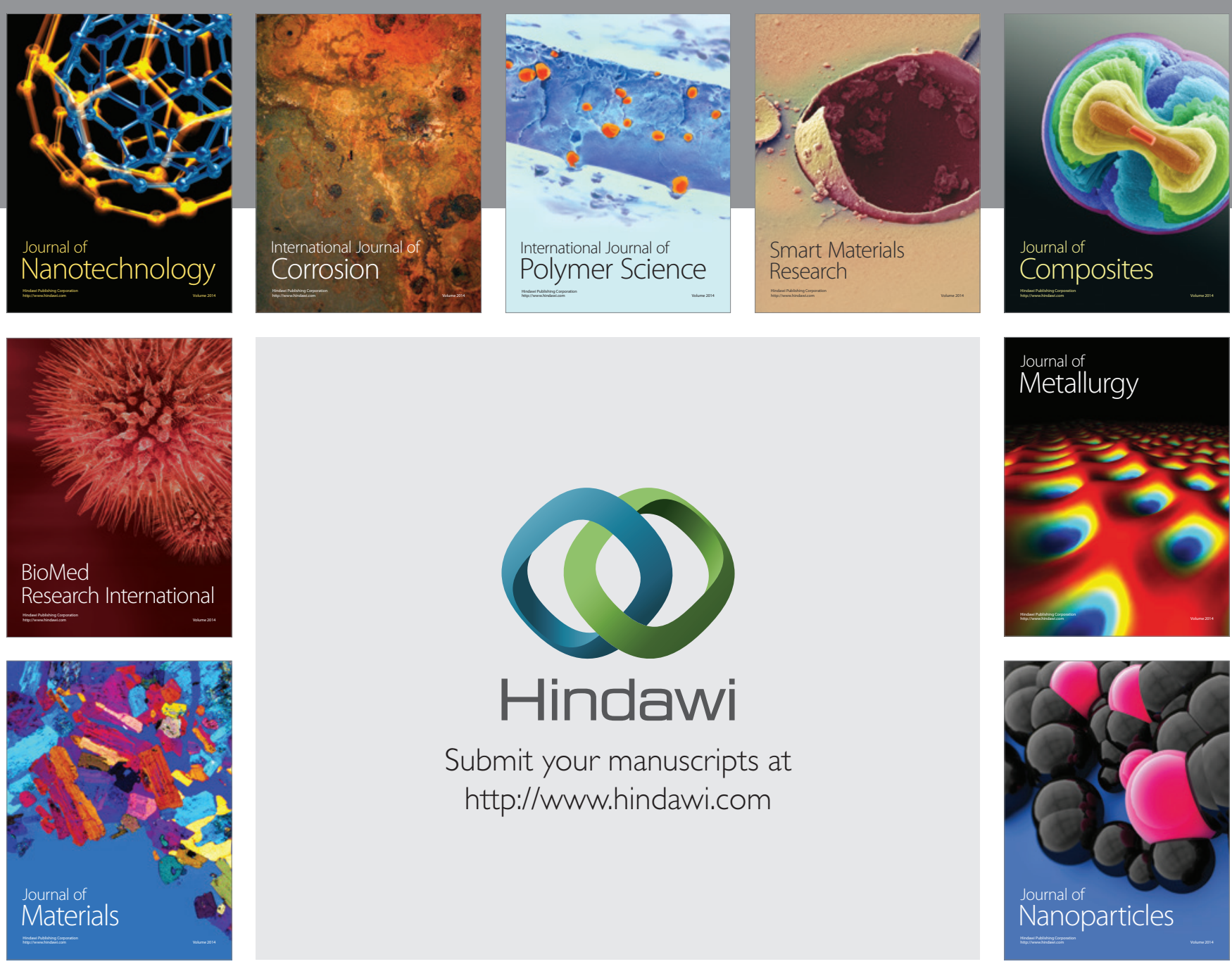

Submit your manuscripts at http://www.hindawi.com
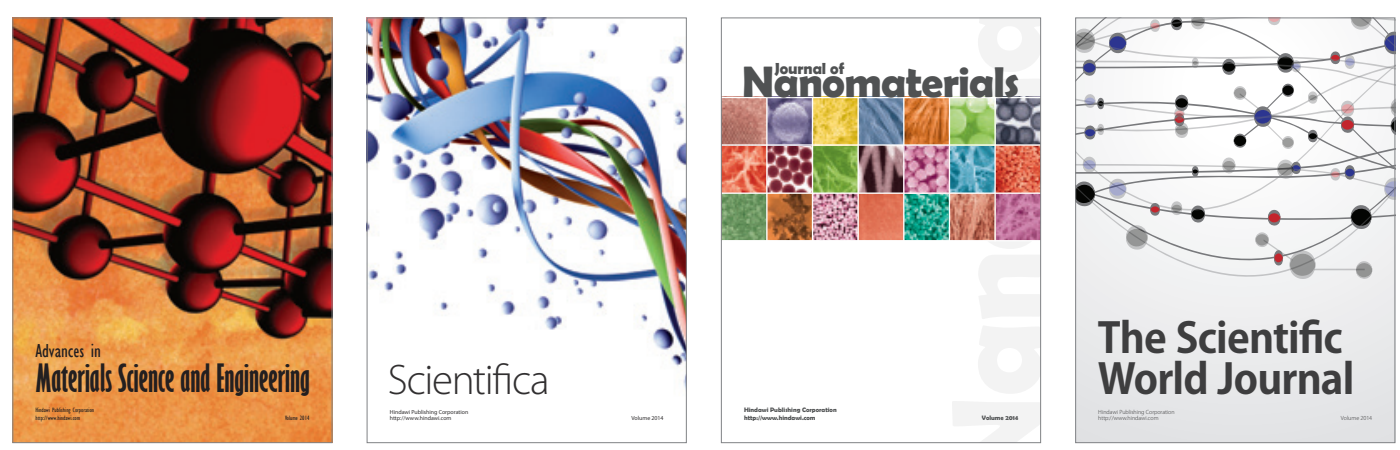

\section{The Scientific World Journal}
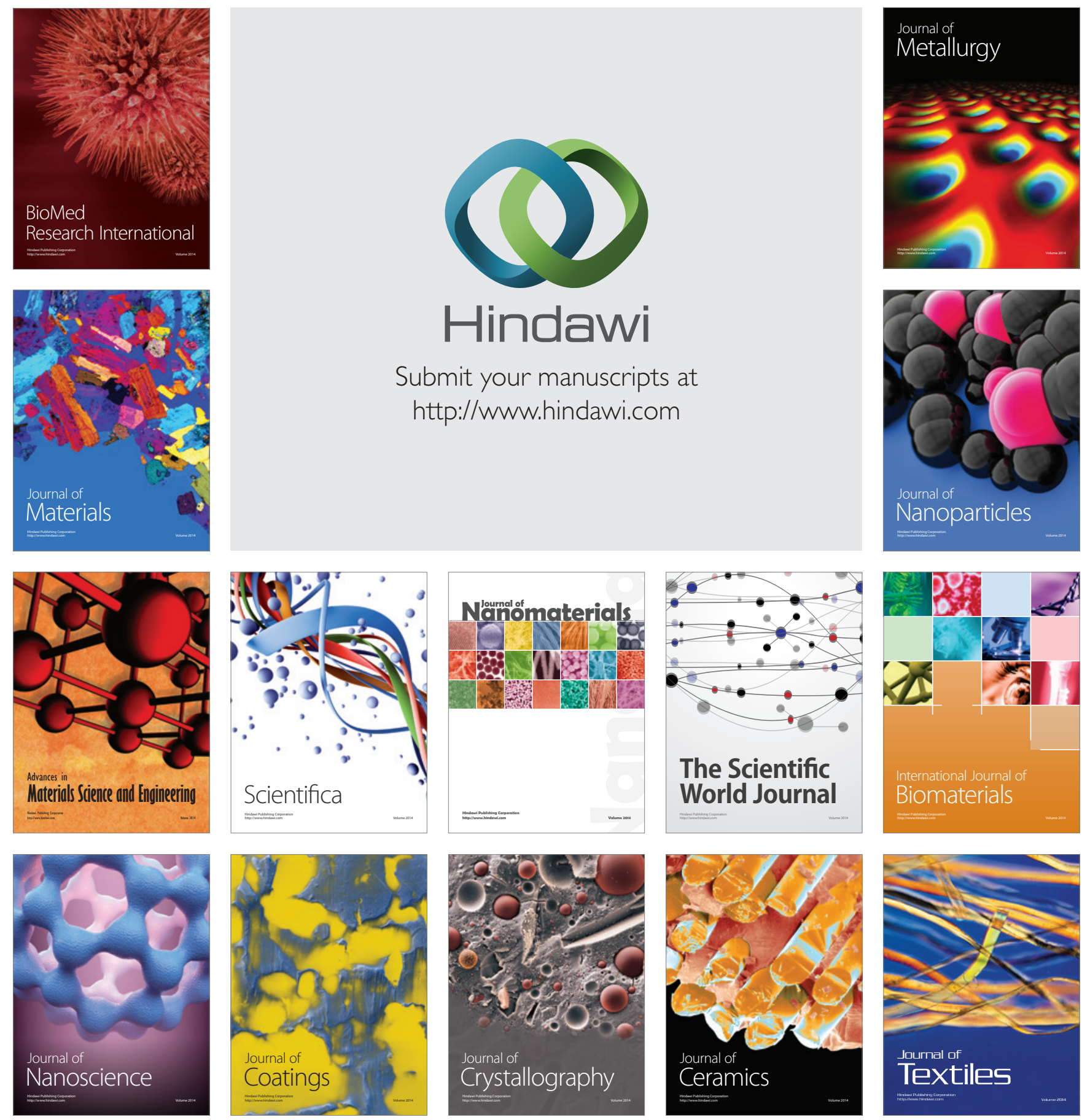